\title{
Integrating Systems Health Management with Adaptive Controls for a Utility-scale Wind Turbine
}

\author{
Susan A. Frost ${ }^{1}$ \\ NASA Ames Research Center, Moffett Field, CA, 94035 \\ Kai Goebel ${ }^{2}$ \\ NASA Ames Research Center, Moffett Field, CA, 94035 \\ Khanh V. Trinh ${ }^{3}$ \\ SGT, Moffett Field, CA, 94035 \\ Mark J. Balas ${ }^{4}$ \\ University of Wyoming, Laramie, WY, 82070 \\ and \\ Alan M. Frost ${ }^{5}$ \\ Consultant, Centennial, WY, 82055
}

\begin{abstract}
Increasing turbine up-time and reducing maintenance costs are key technology drivers for wind turbine operators. Components within wind turbines are subject to considerable stresses due to unpredictable environmental conditions resulting from rapidly changing local dynamics. Systems health management has the aim to assess the state-of-health of components within a wind turbine, to estimate remaining life, and to aid in autonomous decision-making to minimize damage. Advanced adaptive controls can provide the mechanism to enable optimized operations that also provide the enabling technology for Systems Health Management goals. The work reported herein explores the integration of condition monitoring of wind turbine blades with contingency management and adaptive controls. Results are demonstrated using a high fidelity simulator of a utility-scale wind turbine.
\end{abstract}

\section{Introduction}

Q ystem health monitoring provides useful information on the current state of a system that can be used to improve many of its operational objectives ${ }^{1}$. Growing demand for improving the reliability and survivability of safetycritical aerospace systems has led to the development of prognostics and health management (PHM) and faulttolerant control (FTC) systems. Active FTC techniques that are capable of retaining acceptable performance in the presence of faults are being developed for both inhabited and uninhabited air vehicles ${ }^{2-4}$ and researchers are exploring new paradigms and approaches for integrating PHM with controls ${ }^{5,6}$. Typically, a decision-making component reasons over the system health and the objectives and constraints of the system. For instance, a component could be identified as having a fault that would eventually lead to component failure and system

\footnotetext{
${ }^{1}$ Computer Engineer, Intelligent Systems Division, POB 1, M/S 269-3, AIAA Member

${ }^{2}$ Senior Scientist, Intelligent Systems Division, POB 1, M/S 269-3, AIAA Senior Member

${ }^{3}$ Computer Engineer, NASA Ames, POB 1, M/S 269-3

${ }^{4}$ Department Head, Electrical \& Computer Engineering Dept., AIAA Fellow

${ }^{5}$ Wind Energy Consultant, PO Box 30, 3595 Hwy 130
} 
shutdown. Decision making using prognostic information on the estimated remaining useful life (RUL) of the component along with operational objectives and constraints may result in changes to the operational mode of the system or to the system's controller. In this paper, we propose a method to integrate system health monitoring, decision-making, and adaptive control. The proposed architecture will be demonstrated on a simulation of a wind turbine.

Wind turbines operate in highly turbulent environments resulting in aerodynamic loads that can easily excite turbine structural modes, potentially causing component fatigue and failure. Two key technology drivers for turbine manufacturers are increasing turbine up-time and reducing maintenance costs. The trend in wind turbine design is towards larger, more flexible turbines with lower frequency structural modes that can damage system components if they become excited. Accurate models of the dynamic characteristics of new wind turbines are often not available due to the complexity and expense of the modeling task, making wind turbines ideally suited to adaptive control methods. In previous work, adaptive control using residual mode filters has improved the performance and reduced the effects of low frequency structural modes on turbine operation ${ }^{7}$.

Recent advances in structural health monitoring allows for more accurate assessment of the structural health of a system $^{8}$. This paper will demonstrate structural health monitoring of blades on a high fidelity simulation of an adaptively controlled wind turbine with integrated decision-making. The decision-making component will command controller modifications based on reasoning over the health of the turbine blade. It can be extended to accommodate various operating objectives and constraints such as maintenance schedules, component supply chain, wind forecast, cost of energy (COE), and safety.

\section{Introduction to Wind Energy and Wind Turbines}

Wind energy is an important source of green energy. The US and many other countries have aggressive goals to replace non-renewable electricity sources with wind energy ${ }^{8}$. Additionally, wind turbines can be placed in remote settlements without electrical power transmission grid accessibility, such as rural China, to provide a renewable source of electricity. These are some of the factors that are driving huge demands for wind turbine installations. Original equipment manufacturers (OEMs) have increased the size, and hence power generation capacity, of wind turbines dramatically in recent years. Wind power is proportional to the swept area of the rotor and the cube of wind speed. Hence turbines are being built with much longer blades to increase the swept rotor area. Wind speed increases with distance from the ground. So large rotors on tall towers means more energy captured. Additionally, larger turbines can operate in lower wind conditions more cost-effectively. Even though modern turbines are much larger than previous generation turbines, new materials and manufacturing techniques have enabled the manufacturers to increase turbine size while maintaining the relative return on investment.

Both the rapid pace of the OEMs' development and deployment of ever larger turbines and the entry of new players in the market have not always allowed the reliability of these larger systems to keep pace. Of course, turbines usually come with warranty, but the timeliness of problem resolution can have a significant effect on operators' profits. In some cases, OEMs are providing contracts with turbine up-time guarantees, giving the operator more control over expected expenses and income. In that case, the motivation to apply thorough system health monitoring shifts to the service provider.

A wind farm is an interconnected group of wind turbines located in the same area of land that collectively act as a power plant, supplying electrical power to the transmission grid. The wind farm operator manages the complex problem of controlling the turbines and the power supplied to the grid, in addition to determining maintenance schedules and safe operation of the wind farm? .

Wind turbines are complex aerodynamic electro-mechanical systems that operate in unpredictable and sometimes harsh environments. The turbulent nature of the wind can cause turbines to experience extreme loads. The larger turbines are more flexible, resulting in lower frequency resonant modes that are more easily excited and more destructive to the turbine components. Most utility-scale turbines are variable speed with a gearbox between the low speed shaft that is connected to the rotor hub and the high-speed shaft that connects to the generator. The drive train and gearbox are vulnerable to fatigue and failure. Most modern turbine blades are made from composite materials. Blades can be subject to destructive aerodynamic loads, cyclic loads, icing, insect and debris buildup (resulting in a roughness increase), and coupling of resonant modes. Any of these conditions can damage or contribute to damage progression of the composite material ${ }^{10}$. The power electronics of wind turbines are also vulnerable to several types of faults. There are many other failure modes for turbines and their components not mentioned here.

Wind turbine operation is divided into several different regions. Region 1 represents the wind speeds below which the turbine does not operate. The wind speed at the start of Region 2 is called the cut-in wind speed. Rated 
wind speed is the velocity at which maximum power output, or rated power, of a wind turbine is achieved. Region 3 starts at the rated wind speed and extends to the cut-out wind speed, which marks the start of Region 4.

Turbines operating in Region 2 use generator torque to maximize energy capture. In Region 3, the turbine rotational speed is maintained constant at the rated speed by pitching the turbine blades. If a wind turbine were allowed to operate in an uncontrolled manner in Region 3, the power output would increase in proportion to the cube of the wind speed, resulting in overheating of the generator and overstress of the power electronics system. An additional goal of operation in Region 3 is to reduce the loads on the turbine due to aerodynamic forces. Multimegawatt turbines typically have a control strategy for the transition region between Region 2 and Region 3, also called Region 2.5. In Region 4, the turbine blades are locked down and the turbine is yawed out of the wind to prevent damage and for safety.

\section{Adaptive Wind Turbine Controller Design and Simulation}

\section{A. Turbine controller formulation}

The controller designed for the wind turbine simulation is an augmented adaptive controller using residual mode filters. The theory for this controller can be found in Refs. 11-14. It is assumed that the plant is well modeled by the linear time invariant (LTI) system:

$$
\left\{\begin{array}{l}
\dot{x}_{p}=A_{p} x_{p}+B_{p} u_{p}+\Gamma_{p} u_{D} \\
y_{p}=C_{p} x_{p} ; x_{p}(0)=x_{0}
\end{array}\right.
$$

where the plant state $x_{p}$ is an $\mathrm{N}_{\mathrm{p}}$-dimensional vector, the control input vector $u_{p}$ is $\mathrm{M}$-dimensional, and the sensor output vector $y_{p}$ is P-dimensional. The disturbance input vector $u_{D}$ is $\mathrm{M}_{\mathrm{D}}$-dimensional and will be thought to come from the Disturbance Generator:

$$
\left\{\begin{array}{l}
u_{D}=\Theta z_{D} \\
z_{D}=L \phi_{D}
\end{array}\right.
$$

where the disturbance state $z_{D}$ is $\mathrm{N}_{\mathrm{D}}$-dimensional, $\phi_{D}$ is a vector of known basis functions for the solution of $u_{D}=\Theta z_{D}$, and $L$ is a matrix of dimension $\mathrm{N}_{\mathrm{D}}$ by $\operatorname{dim}\left(\phi_{D}\right)$. An important aspect of the theory used in this paper is that the form of the disturbance needs to be known, but the amplitude of the disturbance does not need to be known, i.e., the basis functions in $\phi_{D}$ are known, but $(L, \Theta)$ can be unknown. Some examples of common basis functions are step functions, sine functions, and ramp functions. We can represent a step function of unknown amplitude in the form of Eq. (2) as $\phi_{D} \equiv 1$, with $(L, \Theta)$ unknown.

Well known modern control methods assume that the plant and disturbance generator parameter matrices $(A, B, C, \Gamma, \Theta, L, \phi)$ are known. With this knowledge, the Separation Principle of Linear Control Theory can be invoked to arrive at a state-estimator based, linear controller that can suppress disturbances via feedback ${ }^{15}$. In this paper, we will not assume that the plant and disturbance generator parameter matrices $(A, B, C, \Gamma, \Theta)$ are known.

Instead, we will only assume that the disturbance basis functions $\phi_{D}$ from Eq. (2) are known. In many cases, this is not a severe restriction, since the disturbance function is often of known form but unknown amplitude. For example, disturbances caused by wind gusts encountering a turbine can be modeled by step functions and disturbances caused by motors running at constant speeds on flexible structures can be represented by sine functions.

Our control objective will be to cause the output of the plant $y_{p}$ to asymptotically track zero while accommodating disturbances of the form given by the disturbance generator. We define the output error vector as:

$$
e_{y} \equiv y_{p}-0
$$

To achieve the desired control objective, we want 


$$
e_{y} \underset{t \rightarrow \infty}{\longrightarrow} 0
$$

Consider the plant given by Eq. (1) with the disturbance generator given by Eq. (2). The control objective for this system is accomplished by an adaptive control law of the form:

$$
u_{p}=G_{e} e_{y}+G_{D} \phi_{D}
$$

where $G_{e}$ and $G_{D}$ are matrices of the appropriate compatible dimensions defined by the adaptive gain laws:

$$
\left\{\begin{array}{l}
\dot{G}_{e}=-e_{y} e_{y}^{T} h_{e} \\
\dot{G}_{D}=-e_{y} \phi_{D}^{T} h_{D}
\end{array}\right.
$$

and $h_{e}$ and $h_{D}$ are arbitrary, positive definite matrices. In Ref. 11, it was shown that for a controllable, observable LTI plant that is almost strict positive real (ASPR), the adaptive controller specified by Eqs. (5)-(6) produces asymptotic tracking, i.e., $e_{y} \longrightarrow t \rightarrow \infty$, and the adaptive gains $G_{e}$ and $G_{D}$ remain bounded. A system $(A, B, C)$ is ASPR when $C B$ is positive definite and the open-loop system $P(s)=C(s I-A)^{-1} B$ is minimum phase ${ }^{16}$.

In some cases the plant in Eq. (1) does not satisfy the controller's requirement of ASPR. Instead, there maybe be a modal subsystem that inhibits this property. In Ref. 7 and 14, the above control theory was extended to modify the augmented adaptive controller using a Residual Mode Filter (RMF) to compensate for non-minimum phase modal subsystems, or Q-modes. The non-minimum phase modes are those modes that cause the open-loop plant transfer function to be non-minimum phase, i.e., the transfer function has one or more zeros in the right half plane. Here we describe the augmented adaptive controller using Residual Mode Filters. An advantage of RMF augmentation is that it requires no modification to the control laws or controller gains.

The RMF theory assumes that Eq. (1) can be partitioned into the following form:

$$
\left\{\begin{array}{l}
{\left[\begin{array}{c}
\dot{x} \\
\dot{x}_{Q}
\end{array}\right]=\left[\begin{array}{cc}
A & 0 \\
0 & A_{Q}
\end{array}\right]\left[\begin{array}{c}
x \\
x_{Q}
\end{array}\right]+\left[\begin{array}{c}
B \\
B_{Q}
\end{array}\right] u_{p}+\left[\begin{array}{c}
\Gamma \\
\varepsilon \Gamma_{Q}
\end{array}\right] u_{D}} \\
y_{p}=\left[\begin{array}{ll}
C & C_{Q}-\left[\begin{array}{c}
x \\
x_{Q}
\end{array}\right] ; \varepsilon \geq 0
\end{array}\right.
\end{array}\right.
$$

where $\varepsilon$ represents the amount of leakage of the disturbance into the Q-modal system. To simplify notation, define $x_{p} \equiv\left[\begin{array}{c}x \\ x_{Q}\end{array}\right], A_{p}=\left[\begin{array}{cc}A & 0 \\ 0 & A_{Q}\end{array}\right], B_{p}=\left[\begin{array}{c}B \\ B_{Q}\end{array}\right], C_{p}=\left[C C_{Q}\right], \Gamma_{p}=\left[\begin{array}{c}\Gamma \\ \varepsilon \Gamma_{Q}\end{array}\right]$, and use the disturbance generator as given before by Eq. (2). The output tracking error and control objective remain as in Eqs. (3)-(4), i.e., $e_{y} \equiv y_{p} \longrightarrow t \rightarrow \infty$.

However, now only the subsystem $(A, B, C)$ is assumed ASPR rather than the full un-partitioned plant $\left(A_{p}, B_{p}, C_{p}\right)$, and the modal subsystem $\left(A_{Q}, B_{Q}, C_{Q}\right)$ is assumed known and open-loop stable, i.e. $A_{Q}$ is stable. The disturbance input directly affects the modal subsystem by an amount determined by the parameter $\varepsilon$. So, in summary, the actual plant has an ASPR subsystem and a known modal subsystem that is stable but inhibits the property of ASPR for the full plant. Hence, this modal subsystem must be compensated or filtered away.

We define the Residual Mode Filter (RMF):

$$
\left\{\begin{array}{l}
\dot{\hat{x}}_{Q}=A_{Q} \hat{x}_{Q}+B_{Q} u_{p} \\
\hat{y}_{Q}=C_{Q} \hat{x}_{Q}
\end{array}\right.
$$


And the compensated tracking error:

$$
\tilde{e}_{y} \equiv y_{p}-\hat{y}_{Q}
$$

We now augment the adaptive control law with an RMF:

$$
\left\{\begin{array}{l}
u_{p} \equiv G_{e} \tilde{e}_{y}+G_{D} \phi_{D} \\
\tilde{e}_{y} \equiv y_{p}-\hat{y}_{Q} \\
\dot{\hat{x}}_{Q}=A_{Q} \hat{x}_{Q}+B_{Q} u_{p} \\
\hat{y}_{Q}=C_{Q} \hat{x}_{Q}
\end{array}\right.
$$

with modified adaptive gains:

$$
\left\{\begin{array}{l}
\dot{G}_{e}=-\tilde{e}_{y} \tilde{e}_{y}^{T} h_{e} ; h_{e}>0 \\
\dot{G}_{D}=-\tilde{e}_{y} \phi_{D}^{T} h_{D} ; h_{D}>0
\end{array}\right.
$$

It was shown in Ref. 11 that for a plant given by Eq. (7) with $(A, B, C)$ ASPR, $A_{Q}$ stable, $\phi_{D}$ bounded, that the Augmented Adaptive Controller using RMF in Eqs. (10)-(11) produces $e_{y}=y_{p}$ with exponential rate and bounded adaptive gains $\left(G_{e}, G_{D}\right)$. Also, the state error of the Q-modal system is ultimately bounded into a ball whose radius is determined by both the size of $\varepsilon$, which is related to the amount of disturbance leakage into the Qmodes, and the desired rate of convergence, $a$. When there is no leakage of the disturbance into the Q-modes, i.e., $\varepsilon=0$, the convergence of the state error is asymptotic to zero. Next we describe the wind turbine simulation.

\section{B. Wind turbine simulation}

This study uses a simulation of the 2-bladed Controls Advanced Research Turbine (CART2), an upwind, activeyaw, variable-speed horizontal axis wind turbine (HAWT) located at the National Renewable Energy Laboratory's (NREL) National Wind Technology Center (NWTC) in Golden, Colorado ${ }^{17-18}$. The CART2 is used as a test bed to study control algorithms for medium-scale turbines. The pitch system on the CART2 uses electromechanical servos that can pitch the blades up to $\pm 18 \mathrm{deg} / \mathrm{s}$. In Region 3, the CART2 uses a conventional variable-speed approach to maintain rated electrical power, which is $600 \mathrm{~kW}$ at a low-speed shaft [LSS] speed of $41.7 \mathrm{RPM}$ and a high-speed shaft [HSS] speed of 1800 RPM. Power electronics are used to command constant torque from the generator and full-span blade pitch controls the turbine rotational speed. The maximum rotor-speed for the CART2 is $43 \mathrm{rpm}$ (on the low-speed side) or $1856.1 \mathrm{rpm}$ on the generator side. Whenever the rotor-speed reaches this value the turbine shuts down due to an overspeed condition.

The CART2 has been modeled using the Fatigue, Aerodynamics, Structures, and Turbulence Codes (FAST), a well-accepted simulation environment for HAWTs ${ }^{19}$. The FAST code is a comprehensive aeroelastic simulator capable of predicting both the extreme loads and the fatigue loads of two- and three-bladed horizontal axis wind turbines ${ }^{20}$. Wind turbines can be modeled with FAST as a combination of rigid and flexible bodies connected by several degrees of freedom (DOFs) that can be individually enabled or disabled for analysis purposes. Kane's method is used by FAST to set up equations of motion that are solved by numerical integration. FAST computes the nonlinear aerodynamic forces and moments along the turbine blade using the AeroDyn subroutine package ${ }^{21}$. The FAST code with AeroDyn incorporated in the simulator was evaluated in 2005 by Germanischer LloydWindEnergie and found suitable for 'the calculation of onshore wind turbine loads for design and certification ${ }^{, 22}$.

The parametric information for the FAST simulator as we configured it is available from Ref. 19. The control objective is to regulate generator speed at $1800 \mathrm{rpm}$ and to reject wind disturbances using collective blade pitch. The inputs to the FAST plant are generator torque, blade pitch angle, and nacelle yaw. The FAST simulator can be configured to output many different states or measurements of the plant, such as generator speed and low speed shaft 
velocity. In this study, the yaw is assumed fixed, so that the wind inflow is normal to the rotor. In addition, the generator torque is assumed constant in Region 3. Thus collective blade pitch is the only controller output. Turbine rotational speed, measured on the low-speed shaft side of the gearbox, is the only plant output used by the Region 3 controller.

\section{Controller design}

The adaptive pitch controller was designed with the augmented adaptive control using RMF approach described above. The control objective is to regulate generator speed to a specified value and alleviate aerodynamic loads on the turbine. The uniform wind disturbance, without shear, across the rotor disk of a turbine can be modeled as a step disturbance $^{24}$. Hence, to improve controller performance and reduce loads due to changes in wind speed, we design the adaptive collective pitch controller to reject step disturbances of unknown amplitude. The control objectives are accomplished by collective blade pitch.

First, a control law of the form given in Eq. (11) with gains specified in Eq. (10) is used to design the adaptive collective pitch controller. A step function is used as the disturbance generator function, i.e., $\phi_{D}=1$ from (2). Recall that the amplitude of the disturbance function does not need to be known. This adaptive controller was implemented in Simulink ${ }^{\mathrm{TM}}$ for the FAST simulation of the CART2. The adaptive controller gains, $h_{e}$ and $h_{D}$, were tuned to minimize the generator speed error, while keeping the blade pitch rate in a range similar to that of a baseline Proportional Integral (PI) controller. The gains used in the adaptive controller were: $h_{e}=6.5$ and $h_{D}=0.3$.

A linear model of the turbine running in open-loop and trimmed at a wind speed of $18 \mathrm{mps}$ with the generator and drive-train DOFs enabled is created for analysis. The open-loop transfer function of the linearized plant model has two non-minimum phase zeros at $0.0111 \pm 5.499 i$, thus the plant has two non-minimum phase modes. A Residual Mode Filter is designed from the linear model by first converting the linear system to a modal system. The modal system is partitioned into two subsystems, one minimum phase subsystem and a second stable subsystem with two non-minimum phase zeros. The second subsystem contains the Q-modes, so it will be used as the Residual Mode Filter given in Eq. (8) to augment the adaptive controller to remove the plant's non-minimum phase modes. The transfer function for the RMF used in this study is given by $T(s)=\frac{790.52 s-38.32}{s^{2}+0.02 s+430.30}$.

The RMF is placed in a loop around the controller in the Simulink ${ }^{\mathrm{TM}}$ model of the turbine. The controller output is fed to both the plant and the RMF. The RMF output is subtracted from the plant output, which becomes the controller input. This has the effect of removing the modes from the plant output that inhibit the ASPR property. The augmented adaptive controller using RMF was compared in simulation to the baseline PI controller, see fig. 1.

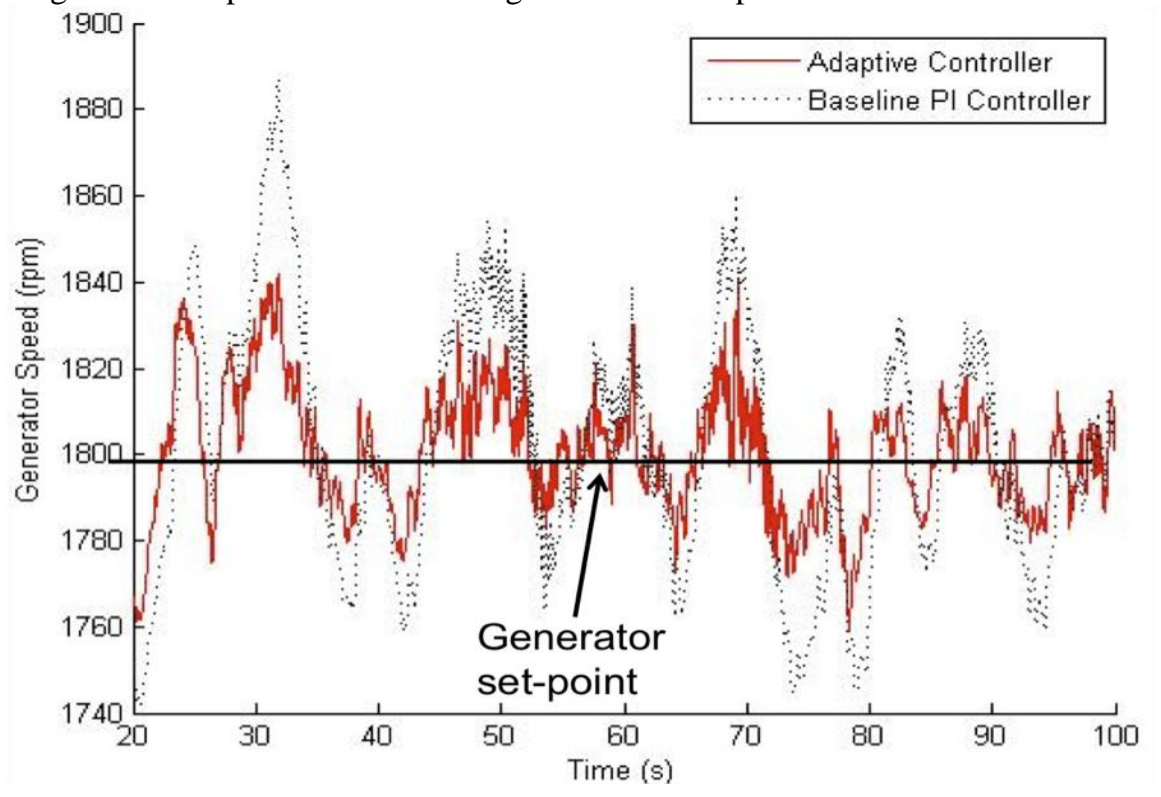

Fig. 1. Comparison of augmented adaptive controller using RMF with baseline PI controller using RMF, with turbulent wind inflow. 


\section{Blade Damage}

In this study, we are interested in exploring optimal wind turbine response in the presence of blade damage. The FAST simulation of the CART2 allows configuration of blade properties at 21 distributed stations along the span. These include sectional mass density, aerodynamic center, flapwise stiffness, edgewise stiffness, chord length, and structural twist. The first and second blade flapwise bending modes and structural damping in percent of critical and the first blade edgewise bending mode and its structural damping in percent of critical are specified in configuration files. Blade mode shapes are represented by sixth-order polynomials that are a function of spanwise position with boundary conditions at the blade root of zero displacement and zero first derivative.

We assume here that blade damage can be represented by a decrease in the spanwise and edgewise stiffness at a blade station. Blade damage such represented includes cracks and delaminations. Changes in blade stiffness result in blade tip deflection changes. A health management system would monitor tip deflection through appropriate sensors (e.g., strain gauges) and, in conjunction with wind speed and blade pitch angle, detect that the deflection has increased. This will further allow for the inference that blade damage is progressing (using appropriate fault mode models and damage propagation models).

A full factorial study with three parameters was performed to determine the spanwise station that was sensitive to changes in stiffness by modifying each blade station one at a time and then measuring the tip displacement during simulation. The study had 8 levels of damage, 7 levels of wind, and 10 levels of blade pitch. Blade configurations where modified with decreased flapwise and edgewise stiffness at a blade station and modified mode shape polynomials that were recomputed and normalized to retain the original blade mode shapes. Results from this preliminary study suggested that the stations most sensitive to stiffness changes were those at mid-span.

Next, a parametric study was carried out to determine the effect of stiffness degradation on the wind turbine blade at blade station 7, which is located 30\% from the blade root (total blade length is 21 meters). The FAST simulation was run without a feedback controller with region 3 wind speeds. The generator torque was held constant at the rated torque. Simulations were run with fixed wind-speed or fixed blade pitch angle, while the residual stiffness at node 7 was decreased. Figure 2 shows a representative plot of the results. Generally, the minimum and maximum deflection of the damaged blade is a function of wind speed and blade pitch. Deflection is also a function of the stiffness, which in turn is a measure for the blade's degree of damage. What is seen in this plot is that the changes of the deflection due to pitch dominate the changes due to changes in stiffness. A similar relationship is observed if the wind speed were changed (not shown in this plot). What this means is that a damage detection tool needs to make sure to factor out the impact of pitch and wind speed to be able to make an inference on the presence of damage in the blade. For the purposes of this example, we use a simple tip displacement threshold logic as a classifier for the detection of blade faults.

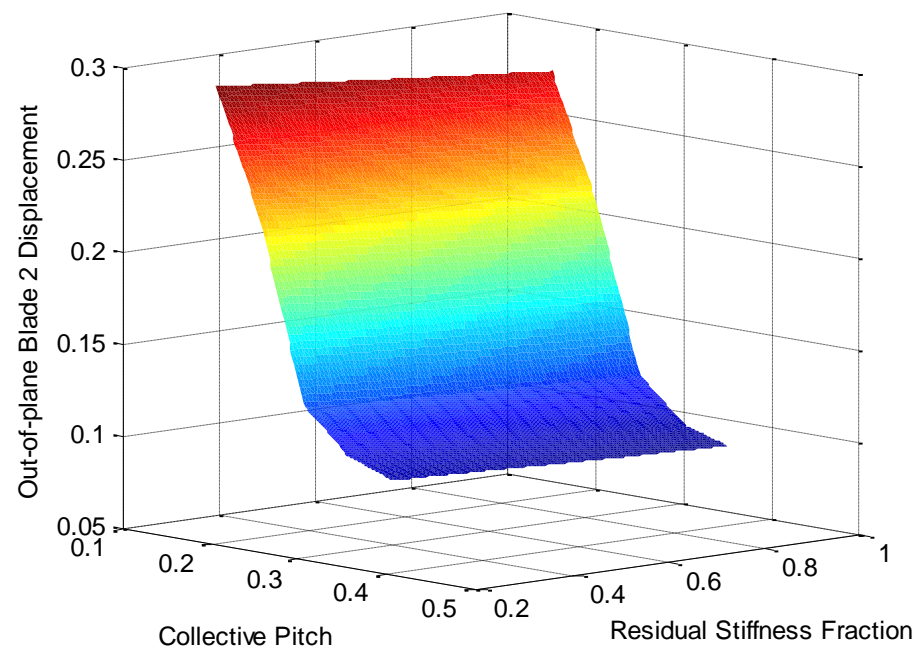

Fig. 2. Delta tip deflection. 


\section{Adaptive controller contingency operation}

We hypothesized that lowering the generator speed set-point, used by the adaptive controller to regulate turbine rotational speed, would reduce the loads on the blade. We ran simulations using the adaptive controller with turbulent wind inflow resulting in primarily Region 3 operation. Figure 3 shows the standard deviation of the out-ofplane tip deflection for the damaged blade for different generator speed operating points. Since the deflection varies considerably with turbulent wind, the standard deviation of the deflection is a more relevant measure to test our hypothesis. Future studies will involve investigating methods to reduce fatigue loads for damaged blades.

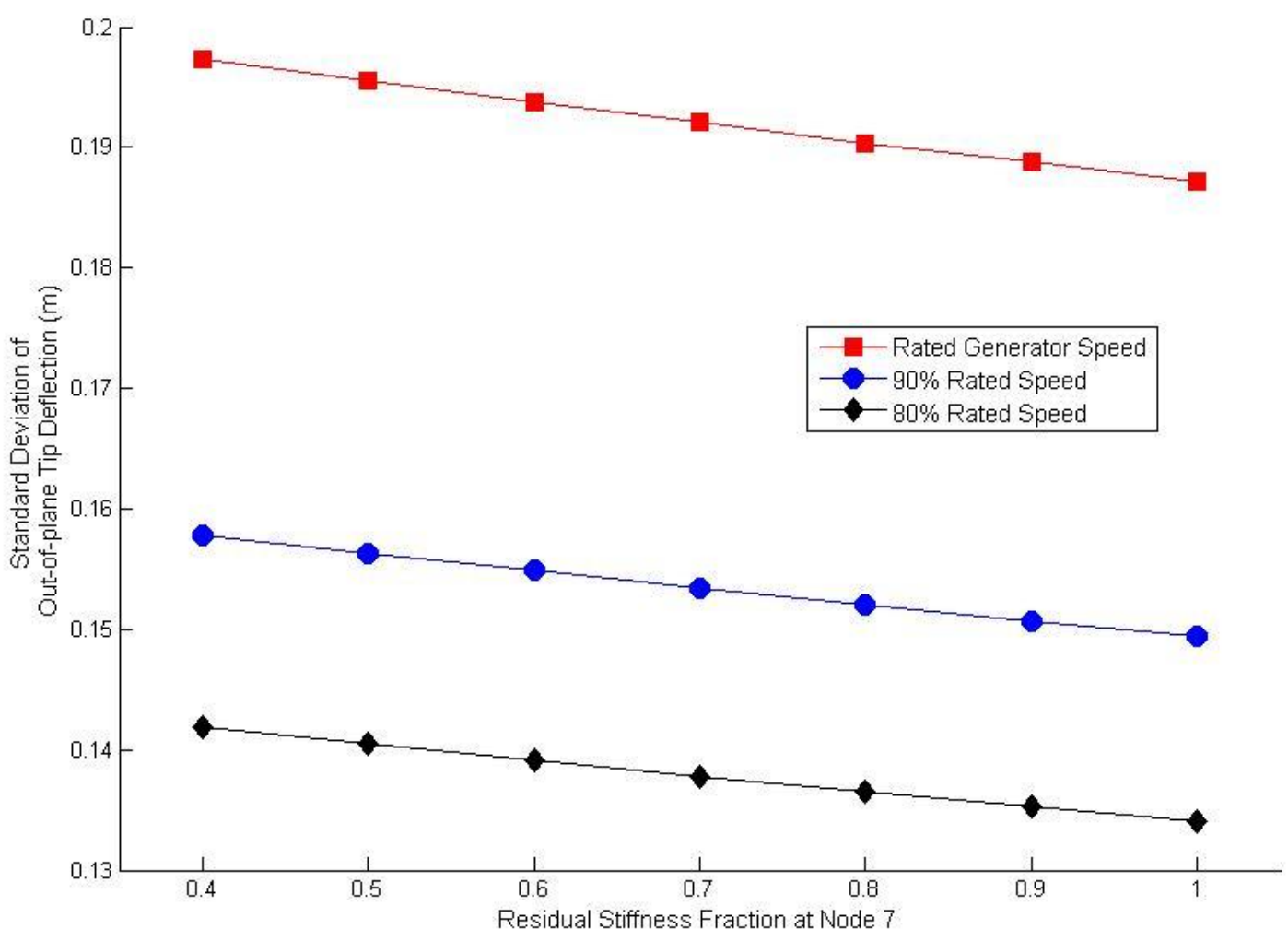

Fig. 3. Standard deviation of out-of-plane tip deflection for different generator speed set-points.

The integration of system health monitoring of wind turbines with controls has the potential for significant payoff when applied to large wind farms or wind parks. The expected power output from a wind farm is a function of the installed name plate capacity of the turbines, e.g., a $2.5 \mathrm{MW}$ turbine, and the expected capacity factor for the wind farm. Contractual obligations to deliver power and the long lead time to replace a damaged turbine, requires wind farm operators to have contingency plans to manage the risk that one or more turbine will suffer damage between scheduled maintenance intervals. If a turbine suffers damage such as a blade delamination, the turbine could potentially operate safely, albeit at a reduced capacity for some period of time. The alternative of shutting a damaged turbine down is the easiest and safest, but potentially leads to lost output and additional costs for the operator. By integrating structural health monitoring with adaptive control algorithms, the operator may be able to run a damaged turbine under a more restricted set of conditions to produce power while the health and safe operation of the turbine is monitored carefully. An optimization algorithm that incorporates wind forecasts, historical data, contractual power output requirements, and maintenance schedules could be integrated with the health monitoring and controls. Such a system would allow the damaged turbine using adaptive control to mitigate the blade stress to generate power under favorable wind conditions when the wind farm power requirements are the highest. The turbine health would be monitored to assess the damage and remaining useful life of the component, to ensure that, 
if the damage progressed, the operating conditions would be further restricted until a decision point where the turbine could no longer be operated safely and it would be shut down.

\section{Conclusion}

We report here on first steps towards integrating systems health monitoring with adaptive controls. In the scenario considered, the adaptive controller receives specific information about damage on the blade and reacts to change the pitch which results in a reduced speed and reduced blade tip deflection. This is meant to decrease the damage propagation. The controller can be tuned to find the optimal trade-off between maximized damage suppression and maximized power generation. Future work will address the assessment of blade health in the presence on nonstationary wind conditions. Future work will also consider a plurality of objectives for wind turbine operations that will allow users to find specific trade-offs between power generation, damage accumulation, maintenance scheduling, cost of repair, etc.

\section{Acknowledgments}

The authors would like to thank Alan Wright at the National Renewable Energy Laboratory and the University of Wyoming Wind Energy Research Center for their support.

\section{References}

${ }^{1}$ Doebling, S.W., Farrar, C.R., Prime, M.B., Shevitz, D.W., "Damage identification and health monitoring of structural and mechanical systems from changes in their vibration characteristics: A literature review," Technical Report LA--13070-MS, Los Alamos National Lab., NM, May 1996.

${ }^{2}$ Y. M. Zhang and J. Jiang, "Bibliographical Review on Reconfigurable Fault-Tolerant Control Systems," in Proc. of the 5th IFAC Symposium on Fault Detection, Supervision and Safety of Technical Processes, Washington, D.C., USA, June 9-11, 2003, pp. 265-276.

${ }^{3}$ D. Shore and M. Bodson, "Flight Testing of a Reconfigurable Control System on an Unmanned Aircraft," AIAA Journal of Guidance, Control, and Dynamics, vol. 28, no. 4, pp. 696-707, 2005.

${ }^{4}$ J. S. Litt, K. J. Parker, and S. Chatterjee, "Adaptive Gas Turbine Engine Control for Deterioration Compensation due to Aging,” Technical Report TM 2003-212607, NASA Glenn, Lewis, OH. 2003.

${ }^{5}$ Liang Tang, Gregory J. Kacprzynski, Kai Goebel, Abhinav Saxena, Bhaskar Saha, and George Vachtsevanos, "Prognosticsenhanced Automated Contingency Management for Advanced Autonomous Systems," International Conference on Prognostics and Health Management, Denver, CO, October 6, 2008 - October 9, 2008.

${ }^{6}$ Farrar, Charles R. and Lieven, Nick A.J., "Damage prognosis: the future of structural health monitoring," Phil. Trans. R. Soc. A, 365(1851), pp. 623-632.

${ }^{7}$ Frost, S.A., Balas, M.J., and Wright, A.D., "Modified Adaptive Control for Region 3 Operation in the Presence of Wind Turbine Structural Modes," 48th AIAA Aerospace Sciences Meeting Including the New Horizons Forum and Aerospace Exposition, January 2010, Orlando, Florida, AIAA 2010-249.

${ }^{8}$ Butterfield, A Sheng, S, Oyague, F. Wind Energy's New Role in Supplying the World's Energy: What Role will Structural Health Monitoring Play?, $7^{\text {th }}$ International Workshop on Structural Health Monitoring, Stanford, CA, Sept 9-11, 2009.

${ }^{9}$ Manwell ,Wind Energy Explained: Theory, Design and Application, $2^{\text {nd }}$ ed., John Wiley \& Sons Ltd, 2009.

${ }^{10}$ M. A. Rumsey and J. A. Paquette, "Structural health monitoring of wind turbine blades," in Proc. SPIE, vol. 6933, Smart Sensor Phenomena, Technology, Networks, and Systems 2008, p. 69330E.

${ }^{11}$ Frost, S.A., Balas, M.J., Wright, A.D.; Direct adaptive control of a utility-scale wind turbine for speed regulation. International Journal of Robust and Nonlinear Control, 2009; 19(1): 59-71.

${ }^{12}$ Balas, M.J.; "Finite-dimensional direct adaptive control for discrete-time infinite-dimensional linear systems," Journal of Mathematical Analysis and Applications 1995; 196(1): 153-171.

${ }^{13}$ Fuentes, R.J., Balas, M.J.; Direct adaptive rejection of persistent disturbances. Journal of Mathematical Analysis and Applications 2000; 251(1): 28-39.

${ }^{14}$ Frost, S.A., Balas, M.J., and Wright, A.D., "Generator Speed Regulation in the Presence of Structural Modes through Adaptive Control using Residual Mode Filters," Special Issue on Modeling and Control of Wind Turbines, Mechatronics, a Journal of IFAC, DOI: 10.1016/j.mechatronics.2011.01.006.

${ }^{15}$ Brogan, W.L., Modern Control Theory, $3^{\text {rd }}$ ed., Prentice-Hall, NJ, 1991, pp. 474-482.

${ }^{16}$ Balas, M.J., Fuentes, R.J.; A Non-orthogonal projection approach to characterization of Almost Positive Real Systems with an application to adaptive control. Proceedings of the American Control Conference, Boston, 2004.

${ }^{17}$ Fingersh, L.J.; Johnson, K.E.; Controls Advanced Research Turbine (CART) Commissioning and baseline data collection. National Renewable Energy Laboratory, NREL/TP-500-32879, Golden, CO, October 2002.

${ }^{18}$ Stol K.A.; Geometry and structural properties for the Controls Advanced Research Turbine (CART) from model tuning. National Renewable Energy Laboratory, NREL/SR-500-32087, Golden, Colorado, Sept. 2004.

${ }^{19}$ National Renewable Energy Lab., NWTC Design Codes (FAST), http://wind.nrel.gov/designcodes/simulators/fast/, National Renewable Energy Lab., Golden, CO, Sept. 2010. 
${ }^{20}$ Jonkman, J.M., Buhl, M.L., FAST user's guide, National Renewable Energy Laboratory, NREL/EL-500-38230, Golden, Colorado, August 2005.

${ }^{21}$ Laino, D.J., Hansen, A.C.; User's guide to the computer software routines AeroDyn interface for ADAMS®. Salt Lake City, Utah: Windward Engineering, LC, September 2001.

${ }^{22}$ Manjock, A.; Evaluation report: Design codes FAST and ADAMS for load calculations of onshore wind turbines. Report No. 72042, Germanischer Lloyd WindEnergie GmbH, Hamburg, Germany, 25 May 2005.

${ }^{23}$ Wright, AD, Balas, MJ, Fingersh, LJ. Testing state-space controls for the controls advanced research turbine. Transactions of the ASME. Journal of Solar Energy Engineering 2006; 128(4): 506-515.

${ }^{24}$ Wright, AD, Balas, MJ. Design of controls to attenuate loads in the controls advanced research turbine. Transactions of the ASME. Journal of Solar Energy Engineering 2004; 126(4): 1083-91. 